\title{
422 - Behavioral Variant Frontotemporal Dementia : an atypical case
}

Filipa Gomes Tavares, Corona Solana, Marco Mota Oliveira Centro Hospitalar Universitário do Algarve

Objective: To present an atypical clinical case and to review the literature on frontotemporal dementia (FTD) focusing on the most frequent psychopathological findings.

Methods: Case report and a non systematic review using databases Pubmed, UpToDate, Medscape, between 2007 to 2020. Keywords: frontotemporal dementia, psychiatry, psychopathology.

Results: FTD is a neurodegenerative syndrome that appears most frequently in the fifth and sixth decades, mostly before the age of 65 . Six to seven years before the diagnosis of FTD, psychiatric disorders such as major depression can appear. The behavioral variant DFT is characterized by symptoms such as disinhibition, apathy or inertia, loss of empathy, hyperorality, persevering behaviors, executive dysfunction, and it is also associated with changes in imaging exams, namely frontal and temporal cortical atrophy, which may affect one or both hemispheres. We present the case of a 66-yearold female patient, accompanied for a long time in psychiatry by conversion and dissociative symptoms, associated with histrionic personality traits. She was hospitalized for deteriorating functioning, pauted by great agitation and maladjusted behavior, alteration of thought and speech, and marked perseverance, as well as periods of space-time disorientation. Analytically, there were no changes, as well as in imaging exams such as CT and MRI. It was performed an electroencephalogram (EEG) that demonstrated diffuse cortical dysfunction.

Discussion/Conclusion: This case is atypical in the DFT pattern, regarding the age of onset and the absence of imaging findings. However, there was alterations in the EEG, which together with the symptomatic presentation point to DFT. This case exemplifies the difficulty in the management of symptoms, whose therapy is purely symptomatic and psychoeducational strategies for the family and caregivers are fundamental. 